\title{
ON A GENERALIZATION OF THE ASSOCIATIVE LAW*
}

BY

\section{ANTON SUSCHKEWITSCH}

1. In my investigations in group theory, I have observed that Lagrange's theorem (that the order of a group is divisible by the order of any subgroup) does not use for its proof the Associative Law in its whole extent; this law can be replaced by a more general postulate, "Postulate A", as I shall call it.

We shall represent our elements by capital italic letters; the operation upon them may be represented by a star $\star$, so that $A \star B$ signifies the result of this operation performed upon $A$ and $B$. A set of elements closed under any operation $\star$ may be called "a group"; this word is thus used in a more general sense than is usual, since the operation $\star$ is arbitrary. The ordinary groups with a special well known operation may be called "classic" to distinguish them from our generalised groups. Sets and groups will be denoted by capital German letters.

Postulate $\mathrm{A}$. In the equation

$$
(X \star A) \star B=X \star C,
$$

the element $C$ depends upon the elements $A$ and $B$ only and not upon $X$. (We suppose here that $X$ can be an arbitrary element of a finite group to which $A, B$ and $C$ belong also.)

The Associative Law is obviously a special case of this Postulate A, viz. if $C=A \star B$.

I have investigated the finite groups that are obtained by replacing the Associative Law in the system of postulates of Frobenius $\dagger$ by Postulate A. I have found the following properties of these groups.

I. Besides our operation $\star$ every group (B) of our type has another operation that will be denoted by a little circle $\circ$ and defined as follows: the equation (1) being given, we write

$$
C=A \circ B .
$$

* Presented to the Society, October 27, 1928; received by the editors in July, 1927.

$\uparrow$ Frobenius (Über endliche Gruppen, Berliner Sitzungsberichte, 1895) defines the classic finite groups by the four following postulates: 1 . The operation that will be considered is uniform (eindeutig) and applicable to any two elements. 2. This operation is uniformly reversible (eindeutig umkehrbar), i.e. from $A B=A C$ or $B A=C A$ it follows that $B=C$. 3. The Associative Law is true for it. 4. The operation is "limited in its effect" (begrenzt in ihrer Wirkung); that signifies the possibility of forming finite groups of our elements. 
It is easy to see, that $B$ is also a group relative to the operation 0 ; we express this fact by writing $B(0)$; (analogously, $(\mathbb{S}(\star))$. I shall prove that (S) (0) is classic.

II. The group $\&(\star)$ has always a right unit (the same for all its elements).

III. If the group \& $(\star)$ has also a single left unit for all its elements (that must necessarily coincide with the right unit), then the Associative Law is true for $\mathbb{B S}(\star)$; in this case $\mathbb{B}(\star)$ is classic and the operations $\star$ and 0 are identical.

It follows that in the systems of postulates of Moore* and Dickson $†$ for the definition of classic groups the Associative Law can be replaced by Postulate A (or its left analogue).

IV. We associate with every element $A$ of our group (S) a substitution

$$
\bar{A} \simeq\left(\begin{array}{c}
X \\
X \star A
\end{array}\right)^{\ddagger}
$$

whereby $X$ runs over all elements of $\mathcal{B}$. I prove that all those substitutions $\bar{A}$ (corresponding to each element $A$ of $(\mathbb{S}$ ) form a substitution group $\bar{\Theta}$ which is obviously classic and simply isomorphic with $B(0)$ : Conversely, all such substitutions $\bar{A}$ form a group only if the Postulate $\mathrm{A}$ is true for $(B)(\star)$.

V. All groups of our type will be obtained from classic groups by making any substitution in the head-line of Cayley's table of a classic group. Moreover, it is sufficient to make only such substitutions as do not alter the unit of the classic group. Such a substitution may be denoted by $\alpha$.

VI. $\mathfrak{S}(\star)$ being any subgroup of $\mathfrak{B}(\star), \mathfrak{S}(0)$ is also a subgroup of (S) (o), i.e. relative to the operation o. The converse is not true. Every subgroup $\mathfrak{S}$ of $\mathbb{B}$ relative to $\circ$ is also a group relative to $\star$, if and only if the substitution $\alpha$, which corresponds to $\rightrightarrows(\star)$, has the following form:

$$
\alpha=\left(\begin{array}{c}
X \\
X^{\imath}
\end{array}\right) \text {, }
$$

the numbers $l$ being relatively prime to the orders of corresponding elements $X$.

2. We shall prove now all the assertions of $\S 1$.

I. The group $(S)(0)$ is obviously uniformly reversible. Again:

$$
[(X \star A) \star B] \star C=[X \star(A \circ B)]_{\star} C=X \star[(A \circ B) \circ C] ;
$$

* Moore, $A$ definition of abstract groups, these Transactions, vol. 3 (1902). (1905).

$\dagger$ Dickson, Definition of a group and a field by independent postulates, these Transactions, vol. 6

$\ddagger$ The sign $\simeq$ signifies that we denote a complicated expression more simply with a single letter. 
and on the other hand

$$
[(X \star A) \star B] \star C=(X \star A) \star(B \circ C)=X \star[A \circ(B \circ C)] ;
$$

and hence the Associative Law is true for $B(0)$.

II. The classic group \&S (o) has always a unit $E$; it is such that

$$
(X \star E) \star A=X \star(E \circ A)=X \star A
$$

and therefore

$$
X \star E=X \text { for every } X ;
$$

$E$ is thus the right unit for $B(\star)$.

III. Let $E$ be a left unit of $\&(\star)$; we have, then,

$$
\left(E_{\star} A\right) \star B=E_{\star}(A \star B)=A \star B ;
$$

and hence by virtue of Postulate $A$ for every element $X$

$$
(X \star A) \star B=X \star(A \star B),
$$

i.e. the Associative Law; hence $B(\star)$ is classic, and $A \circ B=A \star B$.

IV. It follows from (1), by virtue of Postulate $\mathrm{A}$, that $\bar{A} \bar{B}=\bar{C}$; hence $\bar{\Theta}$ is a substitution group simply isomorphic with $B(0)$ (see (2)).

Conversely, let $\$(\star)$ be any finite uniformly reversible group and let $\bar{\Xi}$ be the set of corresponding substitutions, which form also a (classic) group. Let

$$
\overline{A B}=\bar{C}, \quad \text { or } \quad\left(\begin{array}{c}
X \\
X \star A
\end{array}\right)\left(\begin{array}{c}
X \\
X \star B
\end{array}\right)=\left(\begin{array}{c}
X \\
X \star C
\end{array}\right) ;
$$

since

it follows that

$$
\left(\begin{array}{c}
X \\
X \star B
\end{array}\right)=\left(\begin{array}{c}
X \star A \\
(X \star A) \star B
\end{array}\right)
$$

$$
(X \star A) \star B=X \star C
$$

for each element $X$ of $(S)(\star)$; hence Postulate $A$ holds.

$V$. In the head-line of Cayley's table of $\&(\star)$ we make the following substitution:

$$
\alpha \simeq\left(\begin{array}{c}
X \\
E_{\star} X
\end{array}\right)
$$

( $E$ being the right unit of $\&(\star \star))$ ). Let $E_{\star} X=X^{\prime}$. We define the third operation $X$ as follows:

$$
A \star B=A \times B^{\prime} .
$$


The operation $X$ is uniformly reversible and also associative; in fact we have from (1) and (3):

$$
\left(X \times A^{\prime}\right) \times B^{\prime}=X \times C^{\prime},
$$

$C^{\prime}$ depending on $A^{\prime}$ and $B^{\prime}$ only but not on $X$; let $X=E$; then $\left(E \times A^{\prime}\right)$ $\times B^{\prime}=E \times C^{\prime}$; but we have $E \times X^{\prime}=E_{\star} X=X^{\prime}$; hence $C^{\prime}=A^{\prime} \times B^{\prime}$, and (4) gives us the Associative Law for $X$; thus $\&(X)$ is classic. Again it follows from (2) that $\alpha$ gives an isomorphism between $(B)(0)$ and $B(X)$.

Conversely, let $\&(X)$ be now a given classic group; we make in the headline of Cayley's table of $B(X)$ any substitution

$$
\beta \simeq\left(\begin{array}{l}
X \\
\bar{X}
\end{array}\right)
$$

and define a new operation $\star$ as follows:

$$
A \times B=A \star \bar{B} .
$$

The operation $\star$ is obviously uniform and uniformly reversible; the Postulate $A$ is also true for $\star$; in fact, if

we have

$$
(X \star \bar{A}) \star \bar{B}=X \star \bar{C},
$$

$$
(X \star \bar{A}) \star \bar{B}=(X \times A) \times B=X \times(A \times B) ;
$$

and

$$
X \star \bar{C}=X \times C
$$

hence $A \times B=C$ and thus $\bar{C}$ depends upon $\bar{A}$ and $\bar{B}$ only.

$E$ being the unit of $B(X), \bar{E}$ is the right unit for $B(\star)$; we have in fact $A \star \bar{E}=A \times E=A$.

I affirm that we can replace $\beta$ by another substitution $\alpha$, which does not alter $E$, and in this manner define a new operation, say $\square$, so that the group (B) ( $\square$ ) will be simply isomorphic with $\&(\star)$ and have the right unit $E$. We take for $\alpha$

$$
\alpha \simeq\left(\begin{array}{c}
X \\
\bar{X}
\end{array}\right)\left(\begin{array}{c}
\bar{E}_{\star} \bar{X} \\
\bar{X}
\end{array}\right)\left(\begin{array}{c}
\bar{X} \\
X
\end{array}\right)=\left(\begin{array}{c}
X \\
\bar{X}
\end{array}\right)\left(\begin{array}{c}
\bar{E}_{\star} \bar{X} \\
X
\end{array}\right)=\left(\begin{array}{c}
X \\
\bar{X}
\end{array}\right)\left(\begin{array}{c}
\bar{E} \times X \\
X
\end{array}\right) ;
$$

let $\bar{E} \times X^{\prime}=\bar{X}$; we can write then

and so we define

$$
\alpha=\left(\begin{array}{l}
X \\
\bar{X}
\end{array}\right)\left(\begin{array}{c}
\bar{X} \\
X^{\prime}
\end{array}\right)=\left(\begin{array}{l}
X \\
X^{\prime}
\end{array}\right) ;
$$

$$
A \times B=A \square B^{\prime} .
$$


We shall prove that the substitution

$$
\alpha_{1} \simeq\left(\begin{array}{l}
\bar{X} \\
X^{\prime}
\end{array}\right)
$$

gives an isomorphism between the groups $\&(\star)$ and $\&(\square)$. Let

$$
\bar{A} \star \bar{B}=\bar{C} ;
$$

we shall prove that we shall have also

$$
A^{\prime} \square B^{\prime}=C^{\prime} \text {. }
$$

It follows from (5) that $\bar{A} \times B=\bar{C}$; but $\bar{A}=\bar{E} \times A^{\prime}, \bar{C}=\bar{E} \times C^{\prime}$; hence $\left(\bar{E} \times A^{\prime}\right) \times B=\bar{E} \times C^{\prime}$; and since (s) $(X)$ is classic,

$$
\bar{E} \times\left(A^{\prime} \times B\right)=\bar{E} \times C^{\prime} \text {; }
$$

hence $A^{\prime} \times B=C^{\prime}$, and so (6) is established.

VI. Let $\mathfrak{E}=P_{1}+P_{2}+P_{3}+\ldots,{ }^{*} \mathfrak{S}(\star)$ being a subgroup of $\mathfrak{S}(\star)$. Let $\left(X_{\star} P_{\kappa}\right)_{\star} P_{\lambda}=X_{\star} P_{\mu}$; the elements $P_{\kappa}$ and $P_{\lambda}$ of $\mathfrak{S}$ being given, the element $P_{\mu}$ exists also in $\mathfrak{S}$; by virtue of Postulate $\mathrm{A}$ we have $P_{\kappa} \circ P_{\lambda}=P_{\mu}$; hence $\mathfrak{S}(\mathrm{o})$ is also a group.

It follows, hence, that Lagrange's theorem is true for the groups $B(\star)$ of our type.

Let $\mathfrak{S}$ be now a subgroup of $(5)$ relative to $\circ$; we shall analyse the conditions by which $\mathfrak{S}$ is also a group relative to $\star$. Let $\alpha$ be the same substitution as in $\mathrm{V}$, and

$$
\mathfrak{S}^{\prime} \simeq P_{1}^{\prime}+P_{3}^{\prime}+P_{3}^{\prime}+\cdots \text {. }
$$

$\left(P_{1}^{\prime}, P_{2}^{\prime}, P_{3}^{\prime}, \cdots\right.$ are elements in (5) corresponding to $P_{1}, P_{2}, P_{3}, \cdots$, by virtue of $\alpha$.) Since $\alpha$ gives an isomorphism between (S) (O) and $B(X)(X$ being the operation defined by (3)), $\mathfrak{S}^{\prime}(X)$ is also a group (relative to $X$ ).

Let $\mathfrak{S}(\star)$ be also a group; then

$$
P_{\kappa} \star P_{\lambda}=P_{\kappa} \times P_{\lambda}^{\prime} \simeq P_{\mu} .
$$

If $P_{\lambda}^{\prime}$ runs over all elements of $\mathfrak{S}^{\prime}$, then $P_{\mu}$ runs over all elements of $\mathfrak{W}$, and conversely. Hence

$$
P_{\mathfrak{k}} \times \mathfrak{S E}^{\prime}=\mathfrak{S}
$$

(fis each $P_{\kappa}$ of $\left.\mathfrak{S}\right)$. Consequently $\mathfrak{S}$ is one of the partitions of $(\mathbb{S})(X)$ relative to $\mathfrak{E}^{\prime}(X) \dagger$. This condition is obviously also sufficient for $\mathfrak{S}(\star)$ to be a group.

* The sign + signifies that the elements $P_{1}, P_{2}, \cdots$ form a set $\mathfrak{Q}$.

† Hilton, An Introduction to the Theory of Groups of Finite Order, Oxford, 1908, p. 58. 
Since the substitution $\alpha$ does not alter the unit $E$ of $\&(X), \mathfrak{S}$ and $\mathfrak{S}^{\prime}$ must be identically equal to each other, because both of them have a common element $E$.

We shall now analyse the conditions by which every subgroup $\mathfrak{S}(0)$ of (S) (o) is also a group relative to $\star$. Then we must have $\mathfrak{W}^{\prime}=\mathfrak{W}$ (our notation remains as above) for every subgroup $\mathfrak{E}(0)$. We take $\mathfrak{S}^{\prime}=\mathfrak{W}(X)=\{P\}$, a cyclic group, $P$ being an arbitrary element of $B$. Since $\{P\}$ must be also a group relative to $\star$, we have

$$
P^{k} \star P=P^{k} \times P^{l}
$$

consequently for each element $X$ of \& 5 also,

$$
X \star P=X \times P^{\imath} \text {. }
$$

More generally,

$$
X \star P^{\star}=X \times P^{\wedge} .
$$

To every exponent $\alpha$ in (8) there corresponds one and only one exponent $\lambda$ and vice versa. This must be true for each element $P$ of $\left(s ;\right.$ if we take $P_{k}$ instead of $P$, we obtain, in the same manner as in (8),

$$
X \star P^{k \mu}=X \times P^{k \nu} \text {; }
$$

for every $\mu$ there is a definite $\nu$ and vice versa. Let $m$ be the order of $P$, and $d$ the greatest common divisor of $k$ and $m$; then $m / d$ is the order of $P^{k}$ and each exponent $k \mu$ and $k \nu$ in (9) is divisible by $d$. Conversely, if one of the exponents $\kappa, \lambda$ in (8) is prime to $m$, the other is also prime to $m$. Consequently the exponent $l$ in (7) or $\left(7^{\prime}\right)$ must be prime to $m$. Thus $\alpha$ has in this case the following form:

$$
\alpha=\left(\begin{array}{l}
X \\
X^{\imath}
\end{array}\right),
$$

where the numbers $l$ are prime to the orders of corresponding elements $X$. This condition is not only necessary but also sufficient: if it holds, then every cyclic subgroup $\{P\}$ of $B(X)$ is also a group relative to $\star$. But hence every subgroup $\mathfrak{G}(X)$ of $\mathbb{S}(X)$ is also a group relative to $\star$. $Q$ and $P$ being any two elements of $\mathfrak{W}$, we have in fact $Q_{\star} P=Q \times P^{l}$; thus $Q_{\star} P$ belongs also to $\mathfrak{S}$.

We can take, in particular, a substitution $\alpha$ of the following form:

$$
\alpha=\left(\begin{array}{l}
X \\
X^{r}
\end{array}\right),
$$

where $\tau$ is the same for each element $X$ and relatively prime to the order of our group (B). 
3. We shall consider now a special case of Postulate A, that is, however, more general than the Associative Law.

Postulate B. In the equation

$$
(X \star A) \star B=X \star\left(A \star B_{1}\right),
$$

the elements $B$ and $B_{1}$ depend only upon each other; every $B$ is completely defined by the corresponding $B_{1}$, and conversely.

This postulate can be expressed in another form as follows:

Postulate B'. If

$$
A \star B=C \star D
$$

and if $K$ is an arbitrary element, then

$$
A \star(B \star K)=C \star(D \star K) .
$$

We prove first that Postulate $B^{\prime}$ follows from Postulate $B$. Let $R$ be an element such that

$$
A \star(B \star K)=(A \star B) \star R ;
$$

$R$ depends upon $K$ only (by Postulate B). Again it follows from (11) that

$$
(A \star B) \star R=(C \star D) \star R ;
$$

and by Postulate $B$ it follows from (13) that

$$
C \star(D \star K)=(C \star D) \star R ;
$$

hence, from (11), (13), (14), (15) it follows that (12) holds.

Second, we prove that Postulate B follows also from the Postulate $\mathbf{B}^{\prime}$. For that purpose we shall prove the following lemma:

LEMma: If Postulate $\mathrm{B}^{\prime}$ is true for a (uniformly reversible) group (S) $(\star)$, there exists in $\$(\star)$ a right unit (for all elements of $\$(\star)$ ).

If $B$ is a given element, there always exists in $B(\star)$ an element $E$ such that

$$
B \star E=B .
$$

Let $D$ be an arbitrary element of $\&(\star)$; if $A$ is also a given element, there always exists an element $C$, for which

$$
A \star B=C \star D ;
$$

by virtue of Postulate $B^{\prime}$ we have, then,

$$
A \star(B \star E)=C \star(D \star E) ;
$$


and by virtue of (16) and (11) it follows from (17) that $E$ is the right unit for every element $D$.

Assume now that $A \star B=C=C \star E$; in the hypothesis of Postulate $\mathrm{B}^{\prime}$ we have, $K$ being an arbitrary element,

$$
A \star(B \star K)=C \star(E \star K)=(A \star B) \star(E \star K) ;
$$

and thus we have in (13) $E_{\star} K=R$; this shows that Postulate $\mathrm{B}$ holds for our group.

Since the groups with Postulate B form a special case of groups with Postulate A, they can be obtained in the same manner as groups with Postulate A $(\$ 1, V)$. We must now examine what must be the substitution $\alpha(\$ 1, V)$, in order that we may obtain a group $\&(\star)$ with Postulate B. The answer is given by the following theorem:

THEOREM. If the group $B(\star)$ is obtained from the classic group $\mathbb{B}(X)$ by means of the substitution $\alpha$, Postulate $B$ is true for $B(\star)$ if and only if $\alpha$ is an automorphism of the group $B(X)$. In this case $\alpha$ is also an automorphism for (s) $(\star)$, and the operations $\circ$ and $\times$ coincide with each other.

Let $B(\star)$ be a group with Postulate B. The equation (10) gives a dependence of $B$ and $B_{1}$ upon each other; this dependence is given by a substitution, that we denote symbolically by $\left(\begin{array}{l}X \\ X_{1}\end{array}\right)$. Let $A=E$ (the right unit bf (s) $(\star))$ in $(10)$; then

and hence

$$
X \star B=X \star\left(E \star B_{1}\right)
$$

$$
B=E_{\star} B_{1}
$$

Let

we have then

$$
\alpha \simeq\left(\begin{array}{c}
X \\
X^{\prime}
\end{array}\right)
$$

$$
B=E_{\star} B_{1}=E \times\left(B_{1}\right)^{\prime}=\left(B_{1}\right)^{\prime}
$$

and hence

$$
\alpha=\left(\begin{array}{l}
X_{1} \\
X
\end{array}\right)=\left(\begin{array}{l}
X \\
X_{1}
\end{array}\right)^{-1}
$$

Moreover it follows from (10), if we use the notation $A \star B_{1} \simeq C$, that

$$
C=A \circ B=A \star B_{1}=A \times\left(B_{1}\right)^{\prime}=A \times B ;
$$

thus the operations $\circ$ and $\times$ coincide. 
Conversely, suppose that the operations $O$ and $\times$ coincide. Let

we have then

$$
\alpha=\left(\begin{array}{l}
X \\
X^{\prime}
\end{array}\right)=\left(\begin{array}{l}
X_{1} \\
X
\end{array}\right) ;
$$

$$
X \star A=X \times A^{\prime} ;
$$

and

$$
(X \star A) \star B=X \star(A \times B)=X \star\left(A \star B_{1}\right),
$$

and that is Postulate $B$, because $B$ and $B_{1}$ depend only on each other. Again, by (19),

$$
(X \star A) \star B=X \star C=\left(X \times A^{\prime}\right) \times B^{\prime}=X \times C^{\prime} ;
$$

and hence

$$
A \times B=C, \quad A^{\prime} \times B^{\prime}=C^{\prime} ;
$$

this shows us that $\alpha$ is an automorphism of $B(X)$.

Conversely, let $\alpha$ be an automorphism of $B(X)$; then

$$
\begin{aligned}
(X \star A) \star B=\left(X \times A^{\prime}\right) \times B^{\prime} & =X \times\left(A^{\prime} \times B^{\prime}\right) \\
& =X \times{ }_{\star}^{\prime}\left(A_{\star}^{\prime} \times B\right)^{\prime}=X_{\star}(A \times B)=X \star\left(A \star B_{1}\right) ;
\end{aligned}
$$

and thus Postulate B holds.

It remains to prove that $\alpha$ is in this case an automorphism of $\leftrightarrow(\star)$ also. We have in fact

$$
(A \star B)^{\prime}=\left(A^{\prime} \times B^{\prime}\right)^{\prime}=:^{\prime} \times\left(B^{\prime}\right)^{\prime}=A^{\prime} \star B^{\prime} .
$$

4. In the theory of uniformly reversible groups we can consider the operations inverse to the operation of a given group. Since the operation of our group is performed upon two elements (viz. $X_{\star} Y$ ), two inverse operations exist according as the left or the right of these two elements is unknown to us.

If the commutative law is true for our group, such a group has only one inverse operation and only one "inverse group" (i.e. the group relative to the inverse operation). But although a general classic group has two "inverse groups," it has only one inverse operation (abstractly considered), because the properties of the operation of a classic group are "symmetric," i.e. the same on both sides; two "inverse groups" of a classic group are simply isomorphic to each other (if our notations are conveniently chosen); this follows from the fact that a classic group is always "anti-isomorphic" to itself, i.e. there always exists such a substitution $\left(\frac{X}{X}\right)$ of elements of a classic group, that if $A, B$ correspond respectively to $\bar{A}, \bar{B}$, then $A B$ corresponds to $\bar{B} \bar{A}$; we can take, for example, $\bar{X}=X^{-1}$. 
The operation of a finite classic group \& 3 may be denoted by $X$, the two inverse operations by $\triangle$ and $\nabla$; more precisely,

$$
\text { if } A \times B=C \text {, then } C \triangle B=A, C \nabla A=B .
$$

Both inverse groups $\&(\triangle), \&(\nabla)$ are finite and uniformly reversible but not associative. Let us consider what influence the associative law of the operation $\times$ makes on the operations $\triangle$ and $\nabla$. Let $(A \times B) \times C$ $=A \times(B \times C) \simeq R ; A \times B \cong P ; B \times C \cong Q$; then $P \times C=A \times Q=R$. Hence $P \triangle B=A, Q \triangle C=B, R \triangle C=P, R \triangle Q=A$; consequently

$$
(R \triangle C) \triangle B=R \triangle Q, \quad \text { and } B \times C=Q .
$$

[Or $P \nabla A=B, Q \nabla B=C, R \nabla P=C, R \nabla A=Q,(R \nabla A) \nabla B=R \nabla P$, and $P=A \times B$.] This is Postulate $\mathrm{A}$, that is true for the operation $\triangle$ (and for $\nabla)$. But the operations $\Delta$ and $\nabla$ are subject to still another postulate, viz.:

Postulate J. Every element $X$ satisfies the equation

$$
X \triangle X=E \quad(\text { or } \quad X \nabla X=E),
$$

where $E$ is a determined element (the unit of the direct operation $X$ ).

THEOREM 1. A finite uniformly reversible group (S) ( $\star$ ) is an "inverse" to a classic group, if and only if it is subject to the postulates $\mathrm{A}$ and $\mathrm{J}$.

Only one part of this theorem remains for us to prove. Let $\mathbb{B}(\star)$ be subject to the postulates $A$ and $\mathrm{J}$. We use the same notation as before; if $A \star B=C$, then $C \triangle B=A$. We must prove that $\&(\triangle)$ is classic. Obviously the operation $\triangle$ is uniform and uniformly reversible. Again, we have $(X \star A) \star B=X \star C \simeq Z ; C$ depends upon $A$ and $B$ only; let $X \star A \simeq Y$; then $Y \star B=X \star C=Z ; Z \triangle C=X, Z \triangle B=Y, Y \triangle A=X$; thus

$$
(Z \triangle B) \triangle A=Z \triangle C,
$$

which is Postulate A for the operation $\triangle$. It follows from Postulate $\mathrm{J}$, that the group $B(\triangle)$ has a left unit $E$; and hence (see $\S 1$, III) $B(\Delta)$ is classic.

We consider a special case, when our classic group is abelian. We obtain then

THEOREM 2. A finite uniformly reversible group (S) ( $\star$ ) is an "inverse" to an abelian group, if and only if it is subject to the postulates $\mathrm{B}$ and $\mathrm{J}$.

Let $\leftrightarrow(\star)$ be subject to the postulates B and J; by the preceding theorem the inverse group $B(\triangle)$ is classic; it remains for us to show that $B(\triangle)$ is commutative. We have

$$
(X \star A) \star B=X \star\left(A \star B_{1}\right),
$$


$B$ and $B_{1}$ depending only upon each other. Let $A \star B_{1}=C$; then (as in the preceding theorem) $B \triangle A=C, C \triangle B_{1}=A$; hence

$$
B \triangle A \triangle B_{1}=A \text {. }
$$

We write this without brackets, because the Associative Law is true for $\triangle$; (20) is true for each element $A$; we take $A=E$ (unit); then $B \triangle B_{1}=E$; $B_{1}=B^{-1}$; and thus from (20) it follows that $A \triangle B=B \triangle A$; i. e., the Commutative Law holds for $\triangle$.

Conversely, let $B(\triangle)$ be an abelian group; we must prove that $B$ and $B_{1}$ in (10) depend only upon each other. But (20) gives $A \star B^{-1}=B \triangle A=C$; hence $B_{1}=B^{-1}$ in (10), and Postulate B holds for $\star$.

The postulates $B$ and $J$ are characteristic for the operation of division. Thus it is possible, for example, to construct an abstract theory of proportions.

\section{SUPPLEMENT}

Example I. A group with Postulate A but not classic (see Table 1). This group is obtained from the symmetric group of 6 th order by making in the head-line of Cayley's table of this group (see Table 2) the following substitution:

$$
\left(\begin{array}{llllll}
E & A & B & C & D & F \\
E & C & D & A & F & B
\end{array}\right) .
$$

Example II. A group with Postulate B but not classic (see Table 3). This group is obtained from the same symmetric group by making in the head-line of Table 2 the following substitution:

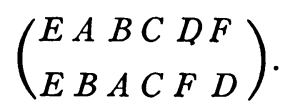

This substitution gives an automorphism of the symmetric group of 6 th order.
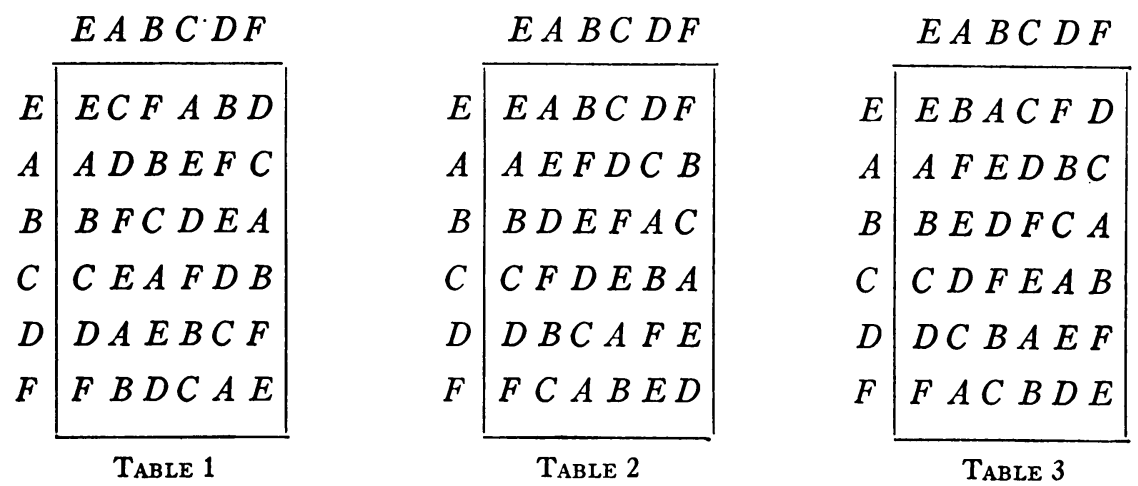

VORONESH UNIVERSITY, VORONESH, RUSSIA 